\title{
Short communication: Milk microbiota profiling on water buffalo with full-length $16 S$ rRNA using nanopore sequencing
}

\author{
Carlotta Catozzi, ${ }^{1 *} \oplus$ Fabrizio Ceciliani, ${ }^{1} \oplus$ Cristina Lecchi, ${ }^{1} \oplus$ Andrea Talenti, ${ }^{2} \odot$ Domenico Vecchio, ${ }^{3}$ \\ Esterina De Carlo, ${ }^{3}$ Carlo Grassi, ${ }^{3}$ Armand Sánchez, ${ }^{4} \odot$ Olga Francino, ${ }^{4} \oplus$ and Anna Cuscó ${ }^{5} \oplus$ \\ ${ }^{1}$ Dipartimento di Medicina Veterinaria, Università degli Studi di Milano, 20133, Italy \\ ${ }^{2}$ The Roslin Institute, University of Edinburgh, Easter Bush Campus, Midlothian, EH25 9RG, United Kingdom \\ ${ }^{3}$ Istituto Zooprofilattico Sperimentale del Mezzogiorno, National Reference Centre for Hygiene and Technologies \\ of Water Buffalo Farming and Productions, 84131 Salerno, Italy \\ ${ }^{4}$ Molecular Genetics Veterinary Service (SVGM), Veterinary School, Universitat Autònoma de Barcelona, 08193 Bellaterra, Barcelona, Spain \\ ${ }^{5}$ Vetgenomics, Ed Eureka, PRUAB, Campus UAB, 08193 Bellaterra, Barcelona, Spain
}

\section{ABSTRACT}

The identification of milk microbial communities in ruminants is relevant for understanding the association between milk microbiota and health status. The most common approach for studying the microbiota is amplifying and sequencing specific hypervariable regions of the $16 S$ rRNA gene using massive sequencing techniques. However, the taxonomic resolution is limited to family and, in some cases, genus level. We aimed to improve taxonomic classification of the water buffalo milk microbiota by amplifying and sequencing the full-length $16 S$ rRNA gene (1,500 bp) using Nanopore sequencing (single-molecule sequencing). When comparing with short-read results, we improved the taxonomic classification, reaching species level. We identified the main microbial agents of subclinical mastitis at the species level that were in accordance with the microbiological culture results. These results confirm the potential of single-molecule sequencing for in-depth analysis of microbial populations in dairy animals.

Key words: milk microbiota, water buffalo, nanopore sequencing

\section{Short Communication}

Unravelling microbiota populations is of pivotal importance in veterinary and animal sciences. The most common method used is targeted sequencing of a specific region of the $16 S \mathrm{rRNA}$ gene. Nevertheless, no consensus about the "best practices" for $16 S$ microbiome studies has been achieved so far (Pollock et al., 2018). For low-biomass samples, such as milk, targeted $16 S$ rRNA gene sequencing approach is one of the best

Received July 30, 2019.

Accepted November 19, 2019.

*Corresponding author: carlotta.catozzi@unimi.it approaches. However, the short length of the targeted $16 S$ regions represents a limitation for identifying taxa below genus level (Gao et al., 2017).

The milk microbiota in ruminants has already been investigated through massive sequencing methods in cows and water buffaloes (Oikonomou et al., 2012; Quigley et al., 2013; McInnis et al., 2015; Catozzi et al., 2017). The association between milk microbiota and health status is particularly relevant for understanding mastitis, by unravelling previously unreported microorganisms potentially related to mammary gland pathogenesis (Hoque et al., 2019) and assessing the milk microbiota recovery after treatment (Catozzi et al., 2019). Microbiological culture technique is considered the gold standard for identifying pathogens in mastitis; however, bacterial DNA is present in culture-negative samples collected from animals with clinical mastitis (Kuehn et al., 2013). The change in milk microbiota during mastitis or lactation stage has already been demonstrated in cows (Lima et al., 2018; Metzger et al., 2018), goats (McInnis et al., 2015), and water buffalo (Catozzi et al., 2017). Given the limited taxonomic resolution of sequencing a short fragment of the $16 S$ rRNA gene, a full-length $16 S r R N A$ gene sequencing by single-molecule sequencers may represent the strategy to reach species-level identification and improve the identification of milk microbiota from healthy and subclinical or clinical mastitis-affected quarters. The Oxford Nanopore Technologies (Oxford, UK) MinION is a portable sequencer, able to generate long reads in real time, allowing direct characterization of the sample. The full-length $16 S$ sequencing using MinION has already been tested on mock communities (Benítez-Páez et al., 2016; Li et al., 2016; Cuscó et al., 2018), pure microbiological culture (Cuscó et al., 2018) and complex communities such as dog skin (Cuscó et al., 2017,2018), the mouse gut (Shin et al., 2016), and the infant gut (Leggett et al., 2017). The aim of this 
experiment was to explore the use of the full-length $16 S$ rRNA gene sequencing by MinION from Oxford Nanopore Technologies (ONT) as a potential approach to improve the taxonomic classification of the bacteria present in water buffalo milk quarters, compared with short-read sequencing analysis.

Twelve water buffalo milk samples were included in this study (see Supplemental File S1 for associated metadata: https://doi.org/10.3168/jds.2019-17359). Six samples were collected from healthy quarters, defined by absence of clinical symptoms, negative microbiological culture, and SCC $<200,000$ cells $/ \mathrm{mL}$ (milk samples 1, 2, 3, 9, 10, and 12), and 6 from subclinical mastitis quarters, characterized by absence of clinical symptoms and positive microbiological culture (milk samples 4, 5, 6, 7, 8, 11). Milk collection, microbiological culture, and SCC analysis were performed as previously described (Catozzi et al., 2017). Briefly, teat ends were treated with a solution of $2 \%$ povidone-iodine (Betadine, Mylan, Italy), and the first 3 streams of milk were discarded to avoid contamination. Milk samples were collected in sterile containers, immediately refrigerated, and delivered to the laboratory, where they were aliquoted. The SCC was measured in milk samples using Integrated Milk Testing Fossomatic 5000 (Foss Analytics, Hillerød, Denmark) apparatus by means of the UNI EN ISO 13366-2:2007 technique for electronic optical fluorometric counters (Hoque et al., 2019). The microbiological culture was applied for each samples: $10 \mu \mathrm{L}$ of milk were cultured using different media, including incubation at $37^{\circ} \mathrm{C}$ for $24 \mathrm{~h}$ in aerobic conditions on Trypticase soy agar (with $5 \%$ sheep blood), MacConkey agar, and Baird-Parker agar, at $37^{\circ} \mathrm{C}$ for $72 \mathrm{~h}$ in aerobic conditions on Prototheca isolation medium, and at $37^{\circ} \mathrm{C}$ for $21 \mathrm{~d}$ in microaerobic conditions on Mycoplasma agar. Gram staining, coagulase, and oxidase tests were performed on cultures with mastitis pathogens.

DNA extraction was carried out and quality assessed as previously reported, starting from $1 \mathrm{~mL}$ of milk (Catozzi et al., 2017). First, V4 $16 S$ amplification (about $250 \mathrm{bp}$ ) was performed using $515 \mathrm{~F}$ and R806 primers, as already described (Catozzi et al., 2019). Briefly, the forward and reverse primers were 5'-CCATCTCATCCCTGCGTGTCTCCGACTC A GN N N N N N N N N N N N N N G ATGTGY CAGCMGCCGCGGTAA-3' (composed of the adapter linker, the key, the barcode, different for each sample, and the forward primer 515F) and 5'-CCTCTCTATGGGCAGTCGGTGATGGACTACNVGGGTWTCTAAT-3' (composed of the adapter linker and the $\mathrm{R} 806$ reverse primer), respectively. We carried out PCR using the Thermo Scientific Phusion Hot Start II HighFidelity DNA polymerase kit (Thermo Fisher Scientific,
Waltham, MA). Each PCR reaction contained nucleasefree water, $5 \times$ Phusion Buffer HF $(5 \mu \mathrm{L})$, dNTP $2 \mathrm{mM}$ $(2.5 \mu \mathrm{L})$, Primer Fw $10 \mu M(1.25 \mu \mathrm{L})$, Primer Rv 10 $\mu M(1.75 \mu \mathrm{L})$, Phusion High-Fidelity Taq Polymerase $2 \mathrm{U} / \mu \mathrm{L}(0.25 \mu \mathrm{L}$ ), and $5 \mathrm{ng}$ of DNA (or $5 \mu \mathrm{L}$ when the concentration was too low). The thermal profile consisted of an initial denaturation of $30 \mathrm{~s}$ at $98^{\circ} \mathrm{C}$, followed by 32 cycles of $15 \mathrm{~s}$ at $98^{\circ} \mathrm{C}, 15 \mathrm{~s}$ at $50^{\circ} \mathrm{C}, 20 \mathrm{~s}$ at $72^{\circ} \mathrm{C}$, followed by a final extension of 7 min at $72^{\circ} \mathrm{C}$. Each PCR plate included a negative template control. The amplicons were sequenced using massive sequencing (Ion Torrent Personal Genome Machine, Thermo Fisher Scientific), as previously reported (Catozzi et al., 2019). In addition to the $V 416 S$ approach using massive sequencing, the full-length $16 S$ rRNA gene (about 1,500 bp) approach was performed using singlemolecule sequencing. A 2-step PCR was carried out as previously described (Cuscó et al., 2018). Briefly, the forward primer 5'-TTTCTGTTGGTGCTGATATTGCAGRGTTTGATYHTGGCTCAG-3', composed of the ONT Universal Tag and the $16 S 27 \mathrm{~F}$ primer, and the reverse primer 5'-ACTTGCCTGTCGCTCTATCTTCTACCTTGTTAYGACTT- 3 ', composed of the ONT Universal Tag and the $16 S$ 1492R primer, covered the region from V1 to V9, as already tested (Cuscó et al., 2017). The ONT Universal Tag is needed for the second PCR, where the barcode is added.

The second PCR was carried out using the Thermo Scientific Phusion Hot Start II High-Fidelity DNA polymerase kit. Each PCR reaction contained nucleasefree water, $5 \times$ Phusion Buffer HF $(5 \mu \mathrm{L})$, dNTPs $2 \mathrm{~m} M$ $(2.5 \mu \mathrm{L})$, Primer Fw $10 \mu M(1 \mu \mathrm{L})$, Primer Rv $10 \mu M$ $(2 \mu \mathrm{L})$, Phusion High-Fidelity Taq Polymerase $2 \mathrm{U} / \mu \mathrm{L}$ $(0.25 \mu \mathrm{L})$, and $5 \mu \mathrm{L}$ of DNA milk samples. One PCR negative control was added, to test contamination of the water or kit reagents. The thermal profile consisted of an initial denaturation of $30 \mathrm{~s}$ at $98^{\circ} \mathrm{C}$, followed by 25 cycles of $15 \mathrm{~s}$ at $98^{\circ} \mathrm{C}, 15 \mathrm{~s}$ at $51^{\circ} \mathrm{C}, 45 \mathrm{~s}$ at $72^{\circ} \mathrm{C}$, and a final extension of $7 \mathrm{~min}$ at $72^{\circ} \mathrm{C}$. DNA purification performed by means of Agencourt AMPure XP beads (Beckman Coulter, Brea, CA) with a $0.5 \times$ concentration; after that, DNA was eluted in $21 \mu \mathrm{L}$ of nuclease-free water; then, quantity of PCR products were determined using Agilent Bioanalyser 2100 (Agilent Technologies, Santa Clara, CA) and Qubit fluorometer (Invitrogen, Carlsbad, CA). A molarity of $0.5 \mathrm{nM}$ of the first PCR product was required for the second PCR, namely barcoding PCR. The quantification of the first PCR was too low for 5 out of 12 samples, as well as for the PCR negative control; for these samples, all volume was used for the second PCR, and the corresponding dilution was assessed for the other samples in a total volume of $20 \mu \mathrm{L}$. The barcoding PCR was carried out using the same kit used before; the PCR mixture, with 
a total of $78 \mu \mathrm{L}$, was composed of nuclease-free water, $5 \times$ Phusion Buffer HF $(20 \mu \mathrm{L})$, dNTPs $2 \mathrm{~m} M(10 \mu \mathrm{L})$, Phusion High-Fidelity Taq Polymerase $2 \mathrm{U} / \mu \mathrm{l}(1 \mu \mathrm{L})$; then the specific barcode $(2 \mu \mathrm{L}$; from PCR Barcoding kit EXP-PBC001, Oxford Nanopore Technologies) and $20 \mu \mathrm{L}$ of DNA from the first PCR were added. The thermal profile consisted of an initial denaturation of $30 \mathrm{~s}$ at $98^{\circ} \mathrm{C}$, followed by 15 cycles of $7 \mathrm{~s}$ at $98^{\circ} \mathrm{C}, 15 \mathrm{~s}$ at $62^{\circ} \mathrm{C}, 45 \mathrm{~s}$ at $72^{\circ} \mathrm{C}$, and a final extension of $7 \mathrm{~min}$ at $72^{\circ} \mathrm{C}$. The library preparation was carried out following the 1D PCR barcoding amplicon protocol, using the Ligation Sequencing Kit 1D (LQK-LSK108 kit, Oxford Nanopore Technologies). We sequenced the full-length $16 S$ rRNA using MinION in a Flow Cell Mk I (R9.4.1, FLO-MIN106 Oxford Nanopore Technologies).

The bioinformatic workflow already reported by Cuscó and colleagues was used to analyze data, with minor modifications (Cuscó et al., 2018). Briefly, MinKNOWN software (version 1.14.1, GUI version 2.1.14, Oxford Nanopore Technologies) was used to run samples. Then, fast5 files were basecalled and demultiplexed using Albacore version 2.3.1 (Oxford Nanopore Technologies). Barcode and adapter removal, in addition to another demultiplexing step, was performed using Porechop (https://github.com/rrwick/Porechop). An extra trimming of 45 bases from each end was carried out, which corresponded to the length of the universal tag and the custom primer. Sequences were selected by size, keeping those from 1,200 and 1,800 bases long. Sequence alignment and mapping to the Greengenes database (DeSantis et al., 2006), previously trimmed for the full-length $16 S$ gene, was carried out using Minimap2 after they were checked for chimeras by means of Yacrd (https://github.com/natir/yacrd; MinION-Minimap2 worflow). After chimera removal, What's In My Pot (WIMP; Juul et al., 2015) workflow on EPI2ME (Oxford Nanopore Technologies), which uses NCBI RefSeq database (https://www.ncbi.nlm.nih .gov/refseq/), was also performed. The data analysis applied after long-read sequencing by MinION included (1) MinION-Minimap2 workflow, as previously reported (Cuscó et al., 2018), using a mapping-based approach; (2) MinION-EPI2ME workflow, which was applied following WIMP workflow on EPI2ME platform (ONT, based on Centrifuge software; Kim et al., 2016). Final results were compared with those from short-read sequencing (Catozzi et al., 2019).

The subclinical mastitis raw short reads were submitted to NCBI under Bioproject accession number SUB4205063 (Bioproject number PRJNA477950). The healthy raw short reads and the raw long reads were submitted to NCBI under Bioproject accession number SUB5522471 (Bioproject number PRJNA534197).
The gel electrophoresis performed before sequencing showed that the expected band at 1,500 bp was present in all samples, except for PCR negative control (Supplemental Figure S1; https://doi.org/10.3168/jds .2019-17359). Unspecific bands around 700 bp were also present in some samples. Quality filtering results are presented in Supplemental Table S1 (https://doi.org/10 .3168/jds.2019-17359). After the first quality procedure using Porechop, more than $90 \%$ of the sequences were kept. The greatest loss of reads was reported after the sequence length trimming between 1,200 and 1,800 bp, resulting, in some cases, in a decrease of more than half of the sequences. The decrease is related to the nonspecific amplicons around 700 bp belonging to the water buffalo genome, demonstrating that the primers anneal to the host genome and should be improved to avoid host DNA amplification. After applying the MinIONMinimap2 workflow, 2 samples from healthy quarters were discarded. Sample 2 was removed for the limited number of sequences, and sample 3 was excluded from the analysis because $80 \%$ of the sequences belonged to the chloroplast taxonomic class.

In subclinical mastitis quarters, we found that longamplicon results were consistent with those obtained by microbiological culture of subclinical mastitis samples. The MinION-EPI2ME procedure allowed the detection of Streptococcus agalactiae as well as Staphylococcus aureus. The MinION-Minimap2 method was able to detect Staph. aureus species but not Strep. agalactiae, even though this species is present in the Greengenes database; Staphylococcus alactolyticus was misrepresented instead of Strep. agalactiae. Greengenes is regarded as one of the best options for V3 V4 short-read data analysis, given the background of its rate of recall (sensitivity) of $93.2 \%$ at family level and $69.2 \%$ at genus level, which is the highest value obtained among other software database comparisons (Almeida et al., 2018). However, the subclinical mastitis samples that were positive for Strep. agalactiae presented divergent results dependent on the method used for analyzing it; EPI2ME found Strep. agalactiae, whereas Minimap2 with the Greengenes database found Staph. alactolyticus. Using BLAST we verified that samples of these sequences were indeed Strep. agalactiae. This wrong result could be linked to the poor representation of Strep. agalactiae in the Greengenes database (2 references) compared with Staph. alactolyticus (223 references) and their high similarity within the $16 S r R N A$ gene (94\%). Samples positive for Staph. aureus were all in accordance with the microbiological culture result, except for sample 4, where the presence of coagulase-negative staphylococci was mistaken for Staph. aureus, which is a coagulase-positive bacterium (Table 1). 
Table 1. Comparison between microbiological culture results and full-length $16 S$ sequencing using MinION-Minimap2 and MinION-EPI2ME workflows (Oxford Nanopore Technologies, Oxford, UK)

\begin{tabular}{|c|c|c|c|}
\hline Sample & Microbiological culture & MinION-Minimap2 & MinION-EPI2ME \\
\hline 5 & Streptococcus agalactiae & $\begin{array}{l}\text { Streptococcus spp. }(24.3 \%) \\
+ \text { Streptococcus alactolyticus }(35 \%)\end{array}$ & $\begin{array}{l}\text { Strep. agalactiae ATCC } 13813(53.2 \%) \\
+ \text { other Streptococcus spp. }(13.3 \%)\end{array}$ \\
\hline 6 & Strep. agalactiae + CNS & $\begin{array}{l}\text { Streptococcus spp. }(53.4 \%) \\
+ \text { Strep. alactolyticus }(30.4 \%)\end{array}$ & $\begin{array}{l}\text { Strep. agalactiae ATCC } 13813 \text { (45.9\%) } \\
\text { + other streptococci }(13.8 \%) \\
\text { + other Staphylococcus spp. }(9.1 \%)\end{array}$ \\
\hline 8 & Staph. aureus & $\begin{array}{l}\text { Staph. aureus }(98.4 \%) \\
+ \text { other Staphylococcus spp. }(0.63 \%)\end{array}$ & $\begin{array}{l}\text { Staph. aureus }(79.6 \%) \\
+ \text { Staph. aureus ssp. anaerobius }(3.4 \%) \\
+ \text { other Staphylococcus spp. }(6 \%)\end{array}$ \\
\hline 11 & Staph. aureus & $\begin{array}{l}\text { Staph. aureus (12.3\%) } \\
\text { + other Staphylococcus spp. (1.6\%) }\end{array}$ & $\begin{array}{l}\text { Staph. aureus }(8.8 \%) \\
+ \text { Staph. aureus ssp. anaerobius }(1.3 \%)\end{array}$ \\
\hline
\end{tabular}

All taxa detected after short-read, MinION-Minimap2, and MinION-EPI2ME workflows are presented in Supplemental File S1 (https://doi.org/10.3168/jds .2019-17359). The 10 most abundant taxa obtained by long-read sequencing are shown in Figure 1. In healthy quarters, most of the species are in low abundance, as detected by both workflows, with the exception of the $37 \%$ of Enterococcus spp. in sample 12 (Enterococcus casselifavus and gallinarum spp. detected by EPI2ME at 11 and $12 \%$, respectively), as shown in Figure 1; only $10 \%$ of this genus was reported using the $V 416 S$ sequencing method. This taxon is generally associated with feces contamination (Gelsomino et al., 2003; Klein, 2003). However, Enterococcus faecalis was already found in bovine healthy milk quarters with SCC of up to 50,000 cells $/ \mathrm{mL}$ (Oikonomou et al., 2014). Moreover, the presence of fecal bacteria in quarter milk samples may be linked to farming practices, including use of bathing pools. Although this practice may decrease animals' thermal stress, it might increase the risk of fecal contamination, as already reported in our previous study of water buffalo microbiota (Catozzi et al., 2017).

The Corynebacterium genus is typically involved in flavor and aroma in cheese and is often present in healthy samples from water buffalo (Catozzi et al., 2017) and cow milk (Kuehn et al., 2013; Quigley et al., 2013; Addis et al., 2016). The present findings demonstrated that Corynebacterium was present in a range from 2.8 to $7.5 \%$ and from 9.5 to $15.4 \%$ after applying MinION-Minimap2 and short-read workflows, respectively. The main representative species, detected by the MinION-EPI2ME method, was Corynebacterium efficiens (Figure 1).

Jeotgalicoccus psychrophilus was detected by both MinION-Minimap2 and MinION-EPI2ME workflows (relative abundance mean of 4.7 and $2.7 \%$, respective- ly) in healthy samples (Supplemental File S1). Short reads were able to detect this taxon only to genus level, as already demonstrated in water buffalo milk (Catozzi et al., 2017). The presence of this bacteria was also confirmed in goat milk (Callon et al., 2007) and the bovine teat canal (Gill et al., 2006) at the species level.

We also investigated whether the $\alpha$ diversity, emphasizing both the evenness (Simpson's index) and the richness components (Shannon's index) of diversity, could be influenced by the type of platform (Ion Torrent vs. MinION machines). Only the subclinical mastitis samples were found to differ significantly between the 2 technologies using the Shannon index $(P=0.01)$, which focuses on the diversity of taxa. This difference was not observed when using the Simpson index, which focuses on the major taxa present in the data set, confirming the substantial equivalency of the 2 platforms when it comes to detection of the major components of the microbiome. In a diversity, only Ion Torrent was able to distinguish samples according to their pathophysiological status (Supplemental Figure S2; https:// doi.org/10.3168/jds.2019-17359). There may be several possible reasons behind this: (1) the lower error rate in Ion Torrent reads allows for a better distinction of genera, whereas the confounding effect of higher error rates in long reads allows a distinction of species only in case of high-quality self-polished reads; or (2) the length of the reads in ONT allows effective detection of species, reducing the number of genera detected as a function of the lower false positives, whereas the reduced length and error rate of Ion Torrent $(\sim 1.5 \%)$ leads to detection of more false positives, resulting in a higher diversity of genera.

The clustering of samples was performed using principal coordinates analysis $(\mathrm{PCoA})$, non-metric 
multidimensional scaling (NMDS) and constrained canonical analysis of principal coordinates (CAP) using the Bray-Curtis distance matrix (Figure 2). The differ- ent methods allow the visualization of the differences between individuals, with $\mathrm{PCoA}$ being the classical multidimensional scaling, NMDS defining the positions
0

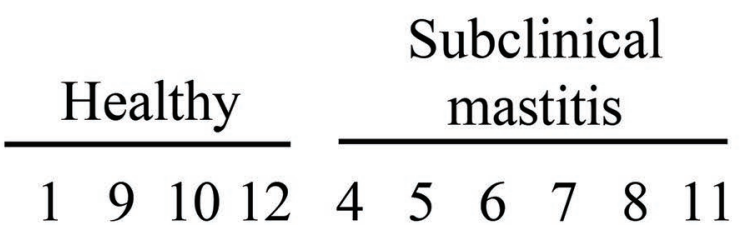
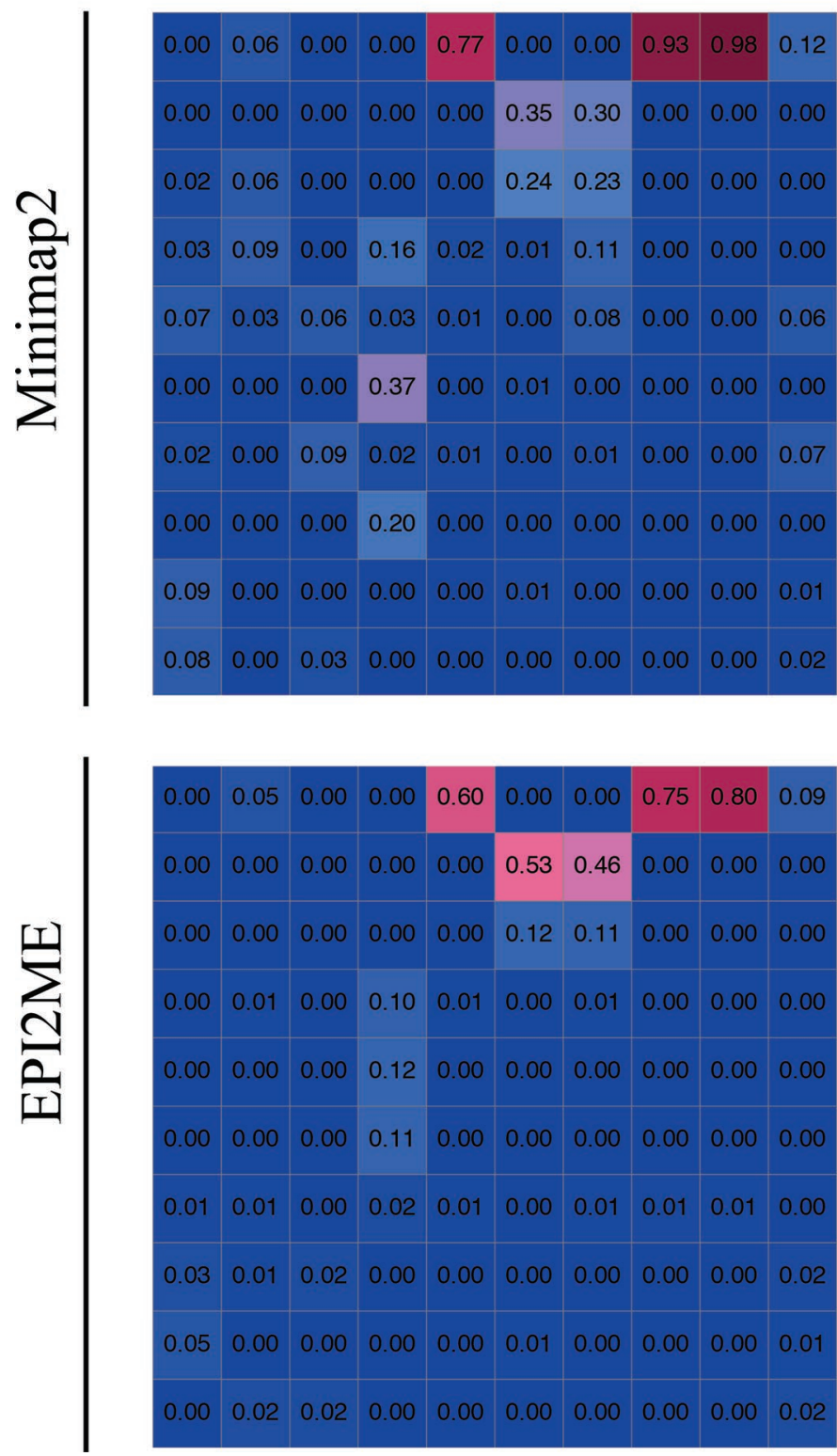

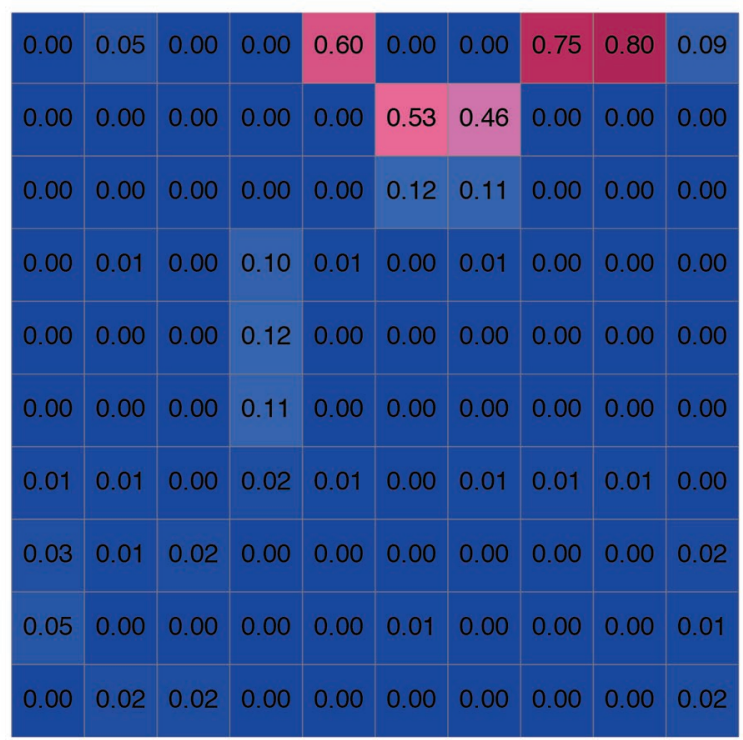

Staphylococcus aureus Streptococcus alactolyticus Streptococcus Staphylococcus Corynebacterium Enterococcus Pseudomonas Stenotrophomonas geniculata 5-7N15 Jeotgalicoccus

Staphylococcus aureus Streptococcus agalactiae Streptococcus dysgalactiae Staphylococcus chromogenes Enterococcus gallinarum Enterococcus casseliflavus Staphylococcus hominis Corynebacterium efficiens Bacteroides plebeius Alkalibacterium iburiense

Figure 1. Heatmap with the relative abundance of the top 10 taxa for healthy and subclinical mastitis samples after using MinIONMinimap2 and MinION-EPI2ME workflows (Oxford Nanopore Technologies, Oxford, UK). Values of relative abundance range from 0 to 1. 
using the nonparametric relationships with the dissimilarities and their positions in a Euclidean space, and CAP decomposing the matrix analyzed before applying the PCoA. All methods show differentiation between both platforms and status of samples. This differentiation is particularly outstanding in CAP, with $15.2 \%$ of the variance linked to the type of platform and $6.6 \%$ connected to the distinction between healthy and sub-
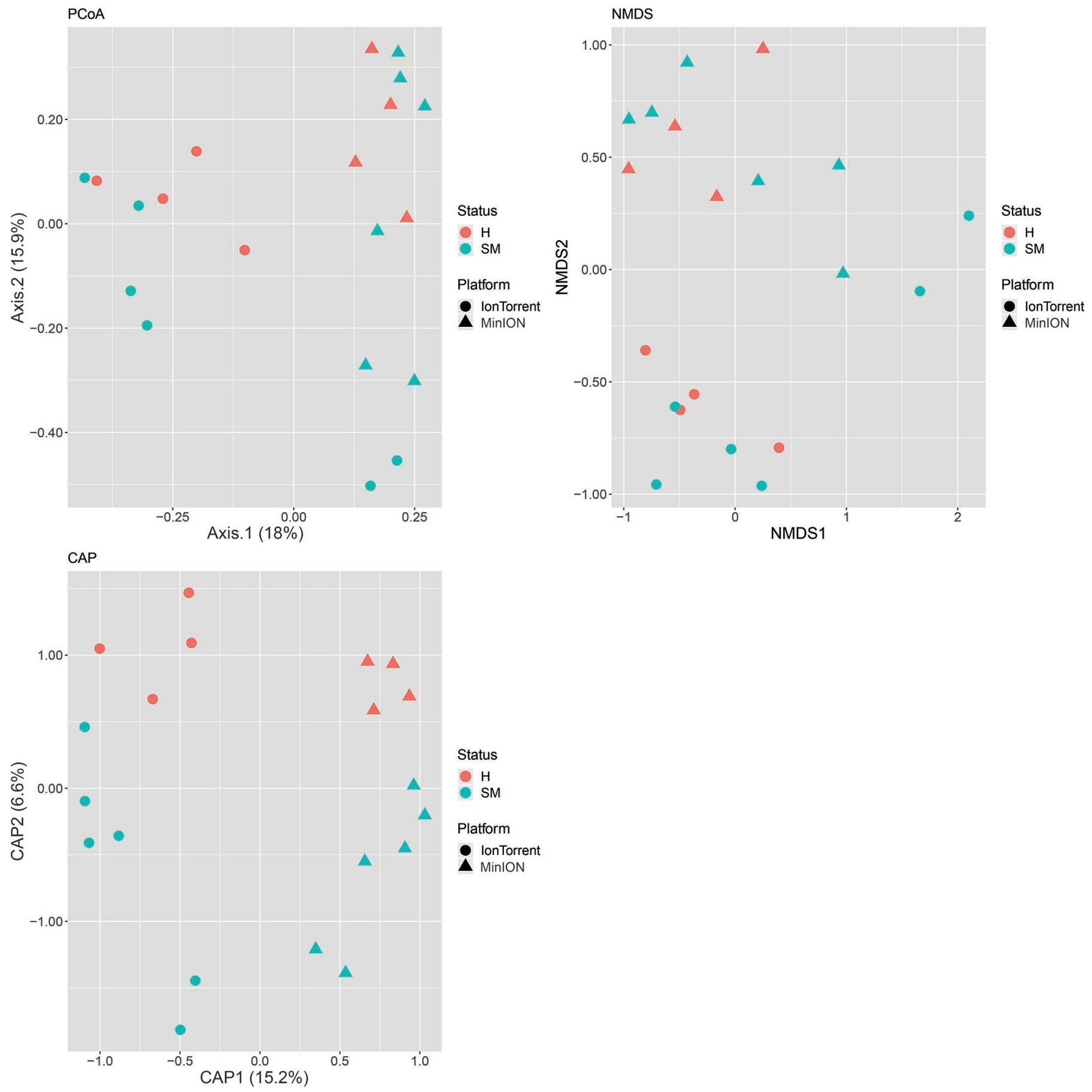

Figure 2. Clustering of healthy $(\mathrm{H})$ and subclinical mastitis $(\mathrm{SM})$ samples by means of principal coordinates analysis (PCoA), non-metric multidimensional scaling (NMDS), and constrained canonical analysis of principal coordinates (CAP) using the Bray-Curtis distance matrix Ion Torrent (Thermo Fisher Scientific, Waltham, MA); MinIon (Oxford Nanopore Technologies, Oxford, UK). 
clinical mastitis samples, suggesting that, despite the effect of methodology, discrimination based on status is still confirmed.

The real-time, portable, and fast method Nanopore MinION could provide a supportive role for microbiological culture, especially in the field, where on-farm culture systems have already been tested for mastitis pathogens in bovine milk (Ganda et al., 2016). Furthermore, milk microbiota characterization up to species level is relevant for identifying possible alterations and dysbiosis, which are not necessarily detected by culture-dependent methods and short-read sequencing. In conclusion, we reported here the results of a pilot study, which would be improved by (1) increasing the number of samples and (2) reassessing the V1 V9 primer choice, as unspecific host amplicons around $700 \mathrm{bp}$ were detected in addition to the expected ones about 1,500 bp level. Furthermore, (3) the full-length $16 S$ sequencing might not be enough to reach species-level identification within some genera. Additionally (4) it would be worthwhile to test other databases or even build up a specific database for milk microbiota analysis. The present study should be regarded as a proof of concept of the application of full-length $16 S \mathrm{rRNA}$ gene sequencing to milk microbiota. Even though further investigation is needed to improve accuracy, the MinION sequencer has demonstrated successful long-read outputs, portability, and real-time analysis, compared with short-read sequencing. These results are preliminary but promising from the clinical point of view. The validation of sequencing results with microbiological culturing is essential to establish a standardized protocol to identify the presence of pathogens in the field. The identification of potential pathogens and resistance genes in milk through metagenomics represents one of the future applications of nanopore technology.

\section{ACKNOWLEDGMENTS}

The authors are grateful to Toby Wilkinson (The Roslin Institute, University of Edinburgh, Easter Bush Campus, Midlothian, United Kingdom) for support in bioinformatic and statistical analyses. This work was supported by Progetti di Ricerca Corrente, AM (Grant number: nr IZS ME 10/13 RC; Ministero della Salute, Rome, Italy). The authors declare no conflict of interest.

\section{REFERENCES}

Addis, M. F., A. Tanca, S. Uzzau, G. Oikonomou, R. C. Bicalho, and P. Moroni. 2016. The bovine milk microbiota: Insights and perspectives from -omics studies. Mol. Biosyst. 12:2359-2372. https:/ /doi.org/10.1039/C6MB00217J.
Almeida, A., A. L. Mitchell, A. Tarkowska, and R. D. Finn. 2018. Benchmarking taxonomic assignments based on 16S rRNA gene profiling of the microbiota from commonly sampled environments. Gigascience 7:1-10. https://doi.org/10.1093/gigascience/giy054.

Benítez-Páez, A., K. J. Portune, and Y. Sanz. 2016. Species-level resolution of $16 \mathrm{~S}$ rRNA gene amplicons sequenced through the Min$\mathrm{ION}^{\mathrm{TM}}$ portable nanopore sequencer. Gigascience 5:4. https://doi .org/10.1186/s13742-016-0111-z.

Callon, C., F. Duthoit, C. Delbès, M. Ferrand, Y. Le Frileux, R. De Crémoux, and M. C. Montel. 2007. Stability of microbial communities in goat milk during a lactation year: Molecular approaches. Syst. Appl. Microbiol. 30:547-560. https://doi.org/10.1016/j .syapm.2007.05.004.

Catozzi, C., A. Cuscó, C. Lecchi, E. De Carlo, D. Vecchio, A. Martucciello, L. D'Angelo, O. Francino, A. Sanchez Bonastre, and F. Ceciliani. 2019. Impact of intramammary inoculation of inactivated Lactobacillus rhamnosus and antibiotics on the milk microbiota of water buffalo with subclinical mastitis. PLoS One 14:e0210204. https://doi.org/10.1371/journal.pone.0210204.

Catozzi, C., A. Sanchez Bonastre, O. Francino, C. Lecchi, E. De Carlo, D. Vecchio, A. Martucciello, P. Fraulo, V. Bronzo, A. Cuscó, S. D'Andreano, and F. Ceciliani. 2017. The microbiota of water buffalo milk during mastitis. PLoS One 12:e0184710. https://doi.org/ 10.1371/journal.pone.0184710.

Cuscó, A., C. Catozzi, J. Vines, A. Sanchez, and O. Francino. 2018. Microbiota profiling with long amplicons using Nanopore sequencing: Full-length 16S rRNA gene and whole rrn operon. F100Reasearch 7:450734. https://doi.org/10.12688/f1000research.16817.1.

Cuscó, A., J. Vines, S. D'Andreano, F. Riva, J. Casellas, A. Sanchez, and O. Francino. 2017. Using MinION to characterize dog skin microbiota through full-length 16S rRNA gene sequencing approach. bioRxiv 167015. https://doi.org/10.1101/167015.

DeSantis, T. Z., P. Hugenholtz, N. Larsen, M. Rojas, E. L. Brodie, K. Keller, T. Huber, D. Dalevi, P. Hu, and G. L. Andersen. 2006. Greengenes, a chimera-checked 16S rRNA gene database and workbench compatible with ARB. Appl. Environ. Microbiol. 72:5069-5072. https://doi.org/10.1128/AEM.03006-05.

Ganda, E. K., R. S. Bisinotto, D. H. Decter, and R. C. Bicalho. 2016. Evaluation of an on-farm culture system (Accumast) for fast identification of milk pathogens associated with clinical mastitis in dairy cows. PLoS One 11:e0155314. https://doi.org/10.1371/ journal.pone.0155314.

Gao, X., H. Lin, K. Revanna, and Q. Dong. 2017. A Bayesian taxonomic classification method for 16S rRNA gene sequences with improved species-level accuracy. BMC Bioinformatics 18:247. https:/ /doi.org/10.1186/s12859-017-1670-4.

Gelsomino, R., M. Vancanneyt, T. M. Cogan, and J. Swings. 2003. Effect of raw-milk cheese consumption on the enterococcal flora of human feces. Appl. Environ. Microbiol. 69:312-319. https://doi .org/10.1128/AEM.69.1.312-319.2003.

Gill, J. J., P. M. Sabour, J. Gong, H. Yu, K. Leslie, and M. W. Griffiths. 2006. Characterization of bacterial populations recovered from the teat canals of lactating dairy and beef cattle by $16 \mathrm{~S}$ rRNA gene sequence analysis. FEMS Microbiol. Ecol. 56:471-481. https://doi.org/10.1111/j.1574-6941.2006.00091.x.

Hoque, M. N., A. Istiaq, R. A. Clement, M. Sultana, K. A. Crandall, A. Z. Siddiki, and M. A. Hossain. 2019. Association of milk microbiome in bovine clinical mastitis and their functional implications in cows in Bangladesh. bioRxiv 591982. https://doi.org/10.1101/ 591982.

Juul, S., F. Izquierdo, A. Hurst, X. Dai, A. Wright, E. Kulesha, R. Pettett, and D. J. Turner. 2015. What's in my pot? Real-time species identification on the MinION ${ }^{\mathrm{TM}}$. bioRxiv 030742. https://doi .org/10.1101/030742.

Kim, D., L. Song, F. P. Breitwieser, and S. L. Salzberg. 2016. Centrifuge: Rapid and sensitive classification of metagenomic sequences. Genome Res. 26:1721-1729. https://doi.org/10.1101/gr.210641 .116.

Klein, G. 2003. Taxonomy, ecology and antibiotic resistance of enterococci from food and the gastro-intestinal tract. Int. J. Food Microbiol. 88:123-131. https://doi.org/10.1016/S0168-1605(03)00175-2. 
Kuehn, J. S., P. J. Gorden, D. Munro, R. Rong, Q. Dong, P. J. Plummer, C. Wang, and G. J. Phillips. 2013. Bacterial community profiling of milk samples as a means to understand culture-negative bovine clinical mastitis. PLoS One 8:e61959. https://doi.org/10 .1371/journal.pone.0061959.

Leggett, R.M., C. Alcon-giner, D. Heavens, S. Caim, T.C. Brook, M. Kujawska, L. Hoyles, P. Clarke, L.J. Hall, and M.D. Clark. 2017. Rapid MinION metagenomic profiling of the preterm infant gut microbiota to aid in pathogen diagnostics. bioRxiv 180406 https:/ /doi.org/10.1101/180406

Li, C., K. R. Chng, E. J. H. Boey, A. H. Q. Ng, A. Wilm, and N. Nagarajan. 2016. INC-Seq: Accurate single molecule reads using nanopore sequencing. Gigascience 5:34. https://doi.org/10.1186/ s13742-016-0140-7.

Lima, S. F., M. L. S. Bicalho, and R. C. Bicalho. 2018. Evaluation of milk sample fractions for characterization of milk microbiota from healthy and clinical mastitis cows. PLoS One 13:e0193671. https:/ /doi.org/10.1371/journal.pone.0193671.

McInnis, E. A., K. M. Kalanetra, D. A. Mills, and E. A. Maga. 2015. Analysis of raw goat milk microbiota: Impact of stage of lactation and lysozyme on microbial diversity. Food Microbiol. 46:121-131. https://doi.org/10.1016/j.fm.2014.07.021.

Metzger, S. A., L. L. Hernandez, J. H. Skarlupka, T. M. Walker, G. Suen, and P. L. Ruegg. 2018. A cohort study of the milk microbiota of healthy and inflamed bovine mammary glands from dryoff through 150 days in milk. Front. Vet. Sci. 5:247. https://doi.org/ 10.3389/fvets.2018.00247.

Oikonomou, G., M. L. Bicalho, E. Meira, R. E. Rossi, C. Foditsch, V. S. Machado, A. G. Teixeira, C. Santisteban, Y. H. Schukken, and R. C. Bicalho. 2014. Microbiota of cow's milk: Distinguishing healthy, sub-clinically and clinically diseased quarters. PLoS One 9:e85904. https://doi.org/10.1371/journal.pone.0085904.

Oikonomou, G., V. S. Machado, C. Santisteban, Y. H. Schukken, and R. C. Bicalho. 2012. Microbial diversity of bovine mastitic milk as described by pyrosequencing of metagenomic 16s rDNA. PLoS One 7:e47671. https://doi.org/10.1371/journal.pone.0047671.

Pollock, J., L. Glendinning, T. Wisedchanwet, and M. Watson. 2018. The madness of microbiome: Attempting to find consensus "best practice" for $16 \mathrm{~S}$ microbiome studies. Appl. Environ. Microbiol. 84:e02627-17. https://doi.org/10.1128/AEM.02627-17.

Quigley, L., O. O'Sullivan, C. Stanton, T. P. Beresford, R. P. Ross, G. F. Fitzgerald, and P. D. Cotter. 2013. The complex microbiota of raw milk. FEMS Microbiol. Rev. 37:664-698. https://doi.org/10 .1111/1574-6976.12030.

Shin, J., S. Lee, M. J. Go, S. Y. Lee, S. C. Kim, C. H. Lee, and B. K. Cho. 2016. Analysis of the mouse gut microbiome using fulllength 16S rRNA amplicon sequencing. Sci. Rep. 6:29681. https:/ /doi.org/10.1038/srep29681.

\section{ORCIDS}

Carlotta Catozzi @ https://orcid.org/0000-0002-1028-1489 Fabrizio Ceciliani 으 https://orcid.org/0000-0002-9199-5757 Cristina Lecchi $\odot$ https://orcid.org/0000-0002-7262-1696 Andrea Talenti @ https://orcid.org/0000-0003-1309-3667 Armand Sánchez @ ittps://orcid.org/0000-0001-9160-1124 Olga Francino ๑ https://orcid.org/0000-0002-9022-3835 Anna Cuscó (i) https://orcid.org/0000-0002-9574-5755 